
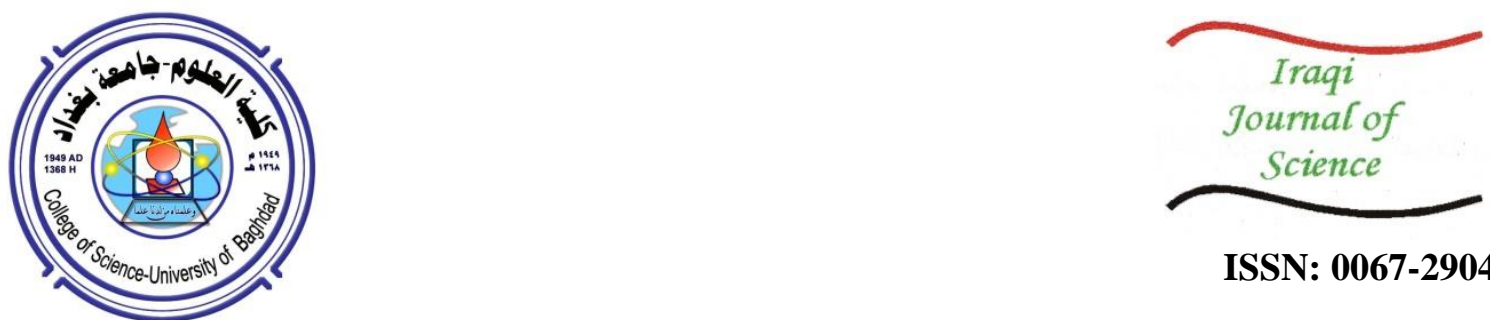

ISSN: 0067-2904

\title{
Electron Scattering from Some $F p$-Shell Nuclei with Inclusion the Effect of Short-Range Correlation
}

\author{
Adel K. Hamoudi, Mahmoud A. Abbas* \\ Department of Physics, College of Science, University of Baghdad, Baghdad, Iraq
}

Received: 23/7/2019 Accepted: 17/12/2019

\begin{abstract}
The effects of short-range correlation on elastic Coulomb (charge) form factors, charge density distributions as well as root mean square charge radii of various $f p$ nuclei (for instance, ${ }^{46,48,50} \mathrm{Ti},{ }^{52,54} \mathrm{Cr},{ }^{56,58} \mathrm{Fe}$, and ${ }^{72,74,76} \mathrm{Ge}$ nuclei) are examined. The one- and two body terms of the cluster expansion together with the singleparticle harmonic oscillator wave functions are utilized. For the purpose of embedding these effects into the formulae of charge density $\rho(r)$ and form factor $F(q)$, we employ the correlation function of Jastrow-type. These formulae depend upon the short-range correlation parameter $\beta$ (which instigates from the Jastrow function) and the oscillator size parameter $b$. Both $\beta$ and $b$ are found by the fitting to the measured elastic form factors. It is noticed that the embedding of shortrange correlation effects into the calculations of $F(q)$ and $\rho(r)$ is a requisite for the achievement of a vital change in the computed results and remarkably vital for the characterization of the measured data.
\end{abstract}

Keywords: short-range correlation effects; electron scattering from atomic nuclei; elastic charge form factors; charge densities; root mean square radii.

الأستطارة الأكترونية المرنة من بعض نوى القشرة-fp مع ادخال تأثير دالة ارتباط المدى القصير

$$
\begin{aligned}
& \text { عادل خلف، محمود عبد القادر * } \\
& \text { قسم الفيزياء، كلية العلوم، جامعة بغداد، بغداد، العراق }
\end{aligned}
$$

الخلاصة

تم دراسة تأثيرات دالة ارتباط المدى التصير على عوامل التثكل الكولومية المرنة وتوزيعات كثافة الثحنة

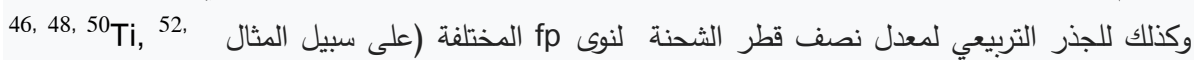
年 الموجة للمتذبذب التوافقي للجسيم المنفرد. لغرض ادخال هذه التأثيرات في صيخ كثافة الثحنة

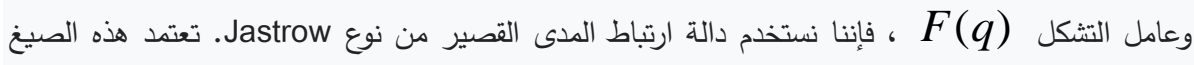

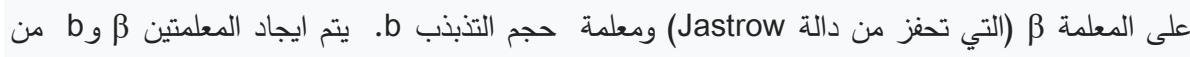
خلال ملائمة عوامل التثكل المرنه المقاسة عمليا. وجدنا بأن ادخال تأثيرات دالة ارتباط المدى القيلة التصير في 


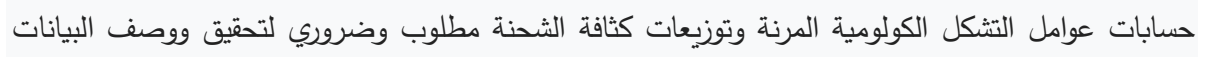

$$
\text { المقاسة عمليا بشكل ملحوظ. }
$$

\section{Introduction}

Estimation of charge densities $\rho(r)$ and elastic charge form factors $F(q)$ of atomic nuclei is an interesting problem [1]. The opportunity of observing this problem is via using the independentparticle model (IPM), where the IPM fails for imitating the measured data at large momentum transfer [2-11]. As a consequence, alteration of single particle potentials (SPP) must be correctly accomplished. Indeed, the repulsion of short-range in the SPP seems proper for light nuclei [12]. For example, by the potential of the harmonic oscillator (HO), the elastic $F(q)$ of light nucleus (such as ${ }^{4} \mathrm{He}$ ) may be well imitated. While for denser nuclei (such as ${ }^{12} \mathrm{C}$ and ${ }^{16} \mathrm{O}$ ), the potentials of the statedependent look are required in despite that the fitting is not well at the high momentum transfer $(q)$ region [12]. An additional method, which is to some extent alike, is the introduction of the short-range correlation (SRC) into the Slater determinant. Numerous studies [4-11, 13-15] were conducted in this tendency through joining light closed shell nuclei in the perspective of the Born approximation.

Czyz and Lesniak [2] were the first to demonstrate that the nature of diffraction in the form factors of ${ }^{4} \mathrm{He}$ may be qualitatively recognized via Jastrow-type correlations [16]. Khana [3] revealed that the embedding of SRC provides an acceptable description of electron scattering data from the nucleus ${ }^{40} \mathrm{Ca}$ and makes predictions for the presentation of the cross section at higher $q$. Atti $[4,5]$ exhibited that electron scattering at larger $q$ looks to provide a sufficient sign for the presence of SRCs in atomic nuclei. Bohigas-Stringari [8] and Dal Ri et al. [9] demonstrated that the one-body form factors offer an adequate check for the existence of SRCs in atomic nuclei. Stoitsov et al. [11] prolonged the model of Jastrow correlations to denser atomic nuclei like ${ }^{16} \mathrm{O},{ }^{36} \mathrm{Ar}$ and ${ }^{40} \mathrm{Ca}$, where this model imitated electron scattering data of these denser nuclei very well.

Massen et al. [13-15], adopting the studies of cluster expansion [17-19], derived a formula for elastic form factor shortened at the two-body part. This formula was utilized for computations of elastic form factor of the closed nuclei ${ }^{4} \mathrm{He},{ }^{16} \mathrm{O}$ and ${ }^{40} \mathrm{Ca}$, as well as using a rough technique of open s-, p-, and sdshell nuclei. Consequently, Massen and Moustakidis [20] accomplished a methodical analysis for the influence of SRC's on s-, p-, and sd-shell nuclei with completely shifting from their technique accomplished in earlier studies [13-15]. Explicit formulae for form factors and densities were derived with the factor cluster expansion and Jastrow correlation functions which introduce the SRCs. These formulae are governed by wave functions of the single-particle and not by those of the relative motion of two-particles as was the situation in our earlier studies [21, 22] and other studies $[4,10,13]$.

There is no full investigation for the influence of short-range correlations on form factors and charge densities of nuclei away from the sd-shell. Thus, in the present investigation, we aim to explore the influence of short-range correlations on elastic charge form factors (ECFF's), $F(q)$, charge density

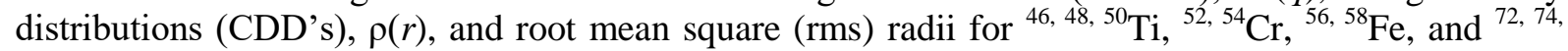
${ }^{76} \mathrm{Ge}$ nuclei. The CDDs and ECFFs formulae depend on the parameters $b$ (the oscillator size parameter) and $\beta$ (the correlation parameter). Here, $b$ and $\beta$ are found by the fit to the measured ECFFs. It is noted that the embedding of SRCs is needed for the attainment of a noticeable change in the computed ECFFs and CDDs as well as for the spectacular illustration of the experimental data.

\section{Theory}

For point nucleon system, the ground state density is given by [20]

$$
\begin{aligned}
\rho_{p}^{g s}(r) & =\frac{\left\langle\Psi\left(r_{1}, r_{2}, \ldots, r_{A}\right)\left|\hat{O}_{r}\right| \Psi\left(r_{1}, r_{2}, \ldots, r_{A}\right)\right\rangle}{\left\langle\Psi\left(r_{1}, r_{2}, \ldots, r_{A}\right) \mid \Psi\left(r_{1}, r_{2}, \ldots, r_{A}\right)\right\rangle} \\
& =N_{D}\left\langle\Psi\left(r_{1}, r_{2}, \ldots, r_{A}\right)\left|\hat{O}_{r}\right| \Psi\left(r_{1}, r_{2}, \ldots, r_{A}\right)\right\rangle \\
& =N_{D}\left\langle\hat{O}_{r}\right\rangle,
\end{aligned}
$$

where $\Psi\left(r_{1}, r_{2}, \ldots, r_{A}\right)$ is the nuclear wave function of the many-particle system, $A$ is the mass number of nuclei, $N_{D}=\left\langle\Psi\left(r_{1}, r_{2}, \ldots, r_{A}\right) \mid \Psi\left(r_{1}, r_{2}, \ldots, r_{A}\right)\right\rangle^{-1}$ is the normalization factor (found by the 
relationship $\left.\frac{4 \pi}{Z} \int_{0}^{\infty} \rho_{p}^{g s}(r) r^{2} d r=1\right)$, and $\hat{O}_{r}$ is the density operator of the one-particle system expressed by

$$
\hat{O}_{r}=\sum_{i=1}^{A} \hat{o}_{r}(i)=\sum_{i=1}^{A} \delta\left(\vec{r}-\vec{r}_{i}\right) .
$$

By means of the cluster expansion, the expectation value of the density operator $\hat{O}_{r}$ is given by

$$
\rho_{p}^{g s}(r)=N_{D}\left\langle\hat{O}_{r}\right\rangle=N_{D}\left\{\left\langle\hat{O}_{r}\right\rangle_{1}+\left\langle\hat{O}_{r}\right\rangle_{2}+\ldots+\left\langle\hat{O}_{r}\right\rangle_{A}\right\} .
$$

The cluster expansion causes the separation of one-, two-,..., A-body correlation effects on the density. In the present analysis, the three-body term and higher terms are ignored. With this approximation, the correlated density $\rho_{p}^{g s}(r)$ is expressed as [20]

$$
\begin{aligned}
\rho_{p}^{g s}(r) & \approx N_{D}\left\{\left\langle\hat{O}_{r}\right\rangle_{1}+\left\langle\hat{O}_{r}\right\rangle_{2}\right\} \\
& \approx N_{D}\left\{\left\langle\hat{O}_{r}\right\rangle_{1}-2 O_{22}(r, \beta)+O_{22}(r, 2 \beta)\right\} .
\end{aligned}
$$

It is evident that the correlated density [eq. (4)] is dependent on the parameter $\beta$ initiated by the correlation of Jastrow-type [16]

$$
F=\prod_{i<j}^{A} f\left(r_{i j}\right)
$$

where the two-body correlation function $f\left(r_{i j}\right)$ is a state-independent function that is given by $f\left(r_{i j}\right)=1-\exp \left[-\beta\left(\vec{r}_{i}-\vec{r}_{j}\right)^{2}\right]$.

Note that eq. (6) satisfies the following two conditions: (1) $f\left(r_{i j}\right) \rightarrow 1$ when $\vec{r}_{i j}=\left|\vec{r}_{i}-\vec{r}_{j}\right|$ is large and (2) $f\left(r_{i j}\right) \rightarrow 0$ when $\vec{r}_{i j} \rightarrow 0$. Consequently, the influence of SRC's becomes essential when the value of $\beta$ is small and vice versa.

In eq. (4), the one-body term $\left\langle\hat{O}_{r}\right\rangle_{1}$ is given by

$$
\begin{aligned}
\left\langle\hat{O}_{r}\right\rangle_{1} & =\sum_{i=1}^{A}\left\langle i\left|\hat{O}_{r}(1)\right| i\right\rangle \\
& =4 \sum_{n l} \eta_{n l}(2 l+1) \frac{1}{4 \pi} \phi_{n l}^{*}(r) \phi_{n l}(r),
\end{aligned}
$$

where $\eta_{n l}$ is the occupation probability and $\phi_{n l}(r)$ is the the radial portion of the single-particle harmonic oscillator wave function of the state $n l$.

In eq. (4), the two-body term $O_{22}(r, z)$ is given by

$$
O_{22}(r, z)=2 \sum_{i<j}^{A}\left\langle i j\left|\hat{o}_{r}(1) g\left(r_{1}, r_{2}, z\right)\right| i j\right\rangle_{a}, \quad(z=\beta, 2 \beta)
$$

where

$$
g\left(r_{1}, r_{2}, z\right)=\exp \left(-z r_{1}^{2}\right) \exp \left(-z r_{2}^{2}\right) \exp \left(2 z r_{1} r_{2} \cos w_{12}\right)
$$

By expanding the $\exp \left(2 z r_{1} r_{2} \cos w_{12}\right)$ in terms of the spherical harmonics [23, 24], the term $O_{22}(r, z)$ is then formed as [20] 


$$
\begin{aligned}
O_{22}(r, z) & =4 \sum_{n_{i} l_{i}, n_{j} l_{j}} \eta_{n_{i} l_{i}} \eta_{n_{j} l_{j}}\left(2 l_{i}+1\right)\left(2 l_{j}+1\right) \\
& \times\left\{4 A_{n_{i} l_{i} n_{j} l_{j}}^{n_{n_{j}} n_{i} l_{i} 0}(r, z)-\sum_{k=0}^{l_{i}+l_{j}}\left\langle l_{i} 0 l_{j} 0 \mid k 0\right\rangle^{2} A_{n_{i} l_{i} n_{j} l_{j}}^{n_{j} l_{j} n_{l_{j}} l_{j}}(r, z)\right\}, \quad(z=\beta, 2 \beta)
\end{aligned}
$$

where

$$
\begin{aligned}
A_{n_{1} l_{2} n_{2} l_{2}}^{n_{3} l_{3} n_{4} l_{4} k}(r, z) & =\frac{1}{4 \pi} \phi_{n_{1} l_{1}}^{*}(r) \phi_{n_{3} l_{3}}(r) \exp \left(-z r^{2}\right) \\
& \times \int_{0}^{\infty} \phi_{n_{2} l_{2}}^{*}\left(r_{2}\right) \phi_{n_{4} l_{4}}\left(r_{2}\right) \exp \left(-z r_{2}^{2}\right) i_{k}\left(2 z r r_{2}\right) r_{2}^{2} d r_{2}
\end{aligned}
$$

and $\left\langle l_{i} 0 l_{j} 0 \mid k 0\right\rangle$ be the coefficient of Clebsch-Gordan.

The CDD $\rho_{c h}^{g s}(r)$ for closed shell (with $N=Z$ ) nuclei can be related to that of the point nucleon system $\rho_{p}^{g s}(r)$ by

$$
\rho_{c h}^{g s}(r)=\frac{1}{2} \rho_{p}^{g s}(r), \quad \text { in }\left(\mathrm{e} . \mathrm{fm}^{-3}\right)
$$

It is significant to point out that eqs. (7) and (10) are initiated for closed shell (with $N=Z$ ) nuclei, where $\eta_{n l}$ is equivalent to 0 or 1 . For isotopes of closed shell nuclei, the correlated CDD's are still given by eqs. (7) and (10) because all the isotopic nuclei own similar $Z$ with the exception that we utilize dissimilar values for parameters $b$ and $\beta$. For open shell nuclei, we also utilize eqs. (7) and (10) but with $0 \leq \eta_{n l} \leq 1$.

The mean square charge radii of the nuclei is defined by

$$
\left\langle r^{2}\right\rangle=\frac{4 \pi}{Z} \int_{0}^{\infty} \rho_{c h}^{g s}(r) r^{4} d r
$$

and the normalzation factor of the $\rho_{c h}^{g s}(r)$ is given by

$$
Z=4 \pi \int_{0}^{\infty} \rho_{c h}^{g s}(r) r^{2} d r
$$

The elastic $F(q)$ of spin zero nuclei can be related to the $\rho_{c h}^{g s}(r)$, where the incident and scattered waves of the electron are characterized by the plane waves. As $\rho_{c h}^{g s}(r)$ is spherically symmetric and real, then the elastic $F(q)$ is just the Fourier transform of $\rho_{c h}^{g s}(r)$, i.e.

$$
F(q)=\frac{4 \pi}{Z} \int_{0}^{\infty} \rho_{c h}^{g s}(r) j_{0}(q r) r^{2} d r
$$

By implanting the corrections [25] of the center of mass $F_{c m}(q)=\exp \left(q^{2} b^{2} / 4 A\right)$ and finite size $F_{f s}(q)=\exp \left(-0.43 q^{2} / 4\right)$ into eq. (15), we have

$$
F(q)=\frac{4 \pi}{Z} \int_{0}^{\infty} \rho_{c h}^{g s}(r) j_{0}(q r) r^{2} d r F_{c m}(q) F_{f s}(q)
$$

It is essential to denote that at $q \rightarrow 0$, the target nucleus is assumed as a point particle and with the benefit of eq. (14), the elastic $F(q)$ [eq. (16)] becomes unity, i.e., $F(q=0)=1$.

\section{Results and discussion}

Computations of elastic $F(q)$ and $\rho_{c h}^{g s}(r)$ of some even fp-shell nuclei (for instance, ${ }^{46,48,50} \mathrm{Ti},{ }^{52}$, ${ }^{54} \mathrm{Cr},{ }^{56,58} \mathrm{Fe}$, and ${ }^{72,74,76} \mathrm{Ge}$ nuclei) are accomplished on the basis of employing the single particle wave functions of the harmonic oscillator with size parameter $b$ together with eqs. (4), (12) and (16). These 
nuclei are consisted of a closed shell core of ${ }^{40} \mathrm{Ca}$ (i.e., filled $1 \mathrm{~s}, 1 \mathrm{p}$ and $2 \mathrm{~s}-1 \mathrm{~d}$ shells) and active particles that move in the orbitals 1f and $2 p$. Here, we suppose that the number of protons in the 1fand 2 p-orbitals is equal to $\left(Z-20-a_{2 p}\right)$ and $a_{2 p}$ protons, respectively. Two cases of calculations are studied, which designate the computations accomplished by the harmonic oscillator wave functions without and with the effect of SRCs. In the first case, the computations are dependent only on the parameter $b$ while in the second case they are dependent on the parameters $b$ and $\beta$. In case 1 (or case 2), the parameters are determined for each nucleus independently by changing $b$ (both $b$ and $\beta$ ) so as to fit the computed elastic form factors to those of experimental ones. The best fit values for $b, \beta$ and for the $\chi^{2}$,

$$
\chi^{2}=\frac{1}{N} \sum_{i=1}^{N}\left[\frac{\left|F_{i}^{\text {cal. }}(q)\right|^{2}-\left|F_{i}^{\text {exp. }}(q)\right|^{2}}{\left|F_{i}^{\text {exp. }}(q)\right|^{2}}\right]^{2},
$$

Table 1-Fitted values for $b$ and $\beta$, and the computed rms charge radii $\left(r_{c h}^{2}\right)^{1 / 2}$ of the one-body, twobody and total densities for various fp-shell nuclei found by the $\chi^{2}$ fit to the experimental ECFFs. Here, case 1 denotes calculations of the one-body term (without SRCs) while case 2 denotes those when

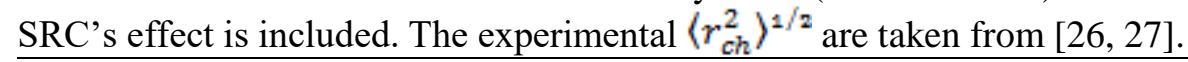

\begin{tabular}{|c|c|c|c|c|c|c|c|c|c|c|}
\hline \multirow{2}{*}{ Case } & \multirow{2}{*}{ Nucleus } & \multicolumn{2}{|c|}{$\begin{array}{l}\text { Proton Occ. } \\
\text { probability }\end{array}$} & \multirow{2}{*}{$\begin{array}{c}b \\
(\mathrm{fm})\end{array}$} & \multirow{2}{*}{$\begin{array}{c}\beta \\
\left(\mathrm{fm}^{-2}\right)\end{array}$} & \multirow{2}{*}{$\chi^{2}$} & \multicolumn{4}{|c|}{$\left\langle r_{c h}^{2}\right)^{1 / 2}(f m)$} \\
\hline & & $\eta_{l f}$ & $\eta_{2 p}$ & & & & $\mathrm{NSR}(\mathrm{HO})$ & SRC & Total & Exp. \\
\hline 1 & ${ }^{46} \mathrm{Ti}$ & 0.143 & 0 & 2.009 & ----- & 0.461 & 3.558 & ----- & 3.558 & 3.564 \\
\hline 2 & ${ }^{46} \mathrm{Ti}$ & & & 1.887 & 2.003 & 0.179 & 3.342 & 1.191 & 3.548 & \\
\hline 1 & ${ }^{48} \mathrm{Ti}$ & & & 2.015 & ----- & 0.387 & 3.568 & ---- & 3.568 & 3.577 \\
\hline 2 & ${ }^{48} \mathrm{Ti}$ & & & 1.891 & 2.007 & 0.175 & 3.349 & 1.187 & 3.553 & ו ו \\
\hline 1 & ${ }^{50} \mathrm{Ti}$ & & & 2.033 & ---- & 0.543 & 3.599 & ---- & 3.599 & 3.567 \\
\hline 2 & ${ }^{50} \mathrm{Ti}$ & & & 1.910 & 2.011 & 0.177 & 3.382 & 1.174 & 3.580 & \\
\hline 1 & ${ }^{52} \mathrm{Cr}$ & 0.286 & 0 & 2.002 & --- & 0.276 & 3.609 & ---- & 3.609 & 3.643 \\
\hline 2 & ${ }^{52} \mathrm{Cr}$ & & & 1.880 & 1.992 & 0.136 & 3.389 & 1.238 & 3.608 & \\
\hline 1 & ${ }^{54} \mathrm{Cr}$ & & & 2.048 & ---- & 0.378 & 3.691 & ---- & 3.691 & 3.689 \\
\hline 2 & ${ }^{54} \mathrm{Cr}$ & & & 1.924 & 2.004 & 0.161 & 3.468 & 1.197 & 3.669 & \\
\hline 1 & ${ }^{56} \mathrm{Fe}$ & 0.429 & 0 & 2.073 & --- & 0.311 & 3.791 & ---- & 3.791 & 3.801 \\
\hline 2 & ${ }^{56} \mathrm{Fe}$ & & & 1.948 & 2.017 & 0.176 & 3.563 & 1.200 & 3.760 & \\
\hline 1 & ${ }^{58} \mathrm{Fe}$ & & & 2.070 & --- & 0.292 & 3.785 & ---- & 3.785 & 3.783 \\
\hline 2 & ${ }^{58} \mathrm{Fe}$ & & & 1.946 & 2.015 & 0.164 & 3.559 & 1.202 & 3.757 & \\
\hline 1 & ${ }^{72} \mathrm{Ge}$ & 0.714 & 0.333 & 2.155 & ---- & 0.473 & 4.062 & ---- & 4.062 & 4.060 \\
\hline 2 & ${ }^{72} \mathrm{Ge}$ & & & 2.026 & 1.932 & 0.271 & 3.823 & 1.354 & 4.055 & \\
\hline 1 & ${ }^{74} \mathrm{Ge}$ & & & 2.157 & ----- & 0.491 & 4.066 & ----- & 4.066 & 4.075 \\
\hline 2 & ${ }^{74} \mathrm{Ge}$ & & & 2.027 & 1.882 & 0.305 & 3.824 & 1.392 & 4.070 & \\
\hline 1 & ${ }^{76} \mathrm{Ge}$ & & & 2.167 & ---- & 0.506 & 4.084 & ---- & 4.084 & 4.081 \\
\hline 2 & ${ }^{76} \mathrm{Ge}$ & & & 2.039 & 1.856 & 0.278 & 3.847 & 1.414 & 4.095 & \\
\hline
\end{tabular}

are shown in Table- 1 . In Table- 1 , the calculated root mean square (rms) charge radii $\left\langle r_{c h}^{2}>^{1 / 2}\right.$ as well as the partaking of SRC's,

$$
<r^{2}>_{2}^{1 / 2}=\sqrt{\left\langle r_{c h}^{2}>-<r_{c h}^{2}>_{1}\right.},
$$

to the $\left\langle r_{c h}^{2}\right\rangle^{1 / 2}$ are also displayed and compared with the corresponding measured data $\left\langle r_{c h}^{2}\right\rangle_{\exp }^{1 / 2}$.

Table- 1 exhibits that the oscillator parameter $b$ (considered in cases 1 and 2) holds the inequality $b$ ( case 1$)>b($ case 2$)$, where the inserting of SRCs leads to raise the relative distance between the nucleons which sequential causes to raise the nuclear size. Accordingly, the parameter $b$, which is related to the nuclear radius (experimentally fixed), must come to be lesser. 
In Figure-1, the ECFF's of ${ }^{46,}{ }^{48,}{ }^{50}$ Ti-isotopes calculated without SRCs (designated by the dashed line) and with SRC's (designated by the solid line) are exposed and compared with the corresponding measured data (designated by the symbols) [28-30]. In these isotopes, the occupation number of proton in $1 \mathrm{f}$ - and $2 \mathrm{p}$-orbitals is assumed to have $\eta_{1 f}=0.143$ and $\eta_{2 p}=0$, correspondingly. The dashed line, in Figures-1(a)-(c), predicts the measured data very well at the momentum transfer region $0 \leq q \leq 1 \mathrm{fm}^{-1}$ and under-predicts them at $q>1 \mathrm{fm}^{-1}$. On the other hand, including the influence of SRCs (the solid line) increases the magnitude of ECFFs at the second maximum region which sequential tends to drive them into the position of accordance with the measured data. Moreover, the first diffraction minimum in addition to the first and second diffraction maxima exposed in the measured data is imitated in the right position by the solid line. Besides, both the presentation and magnitude of ECFF's (shown by the solid line) reveal a well accordance with the measured data.

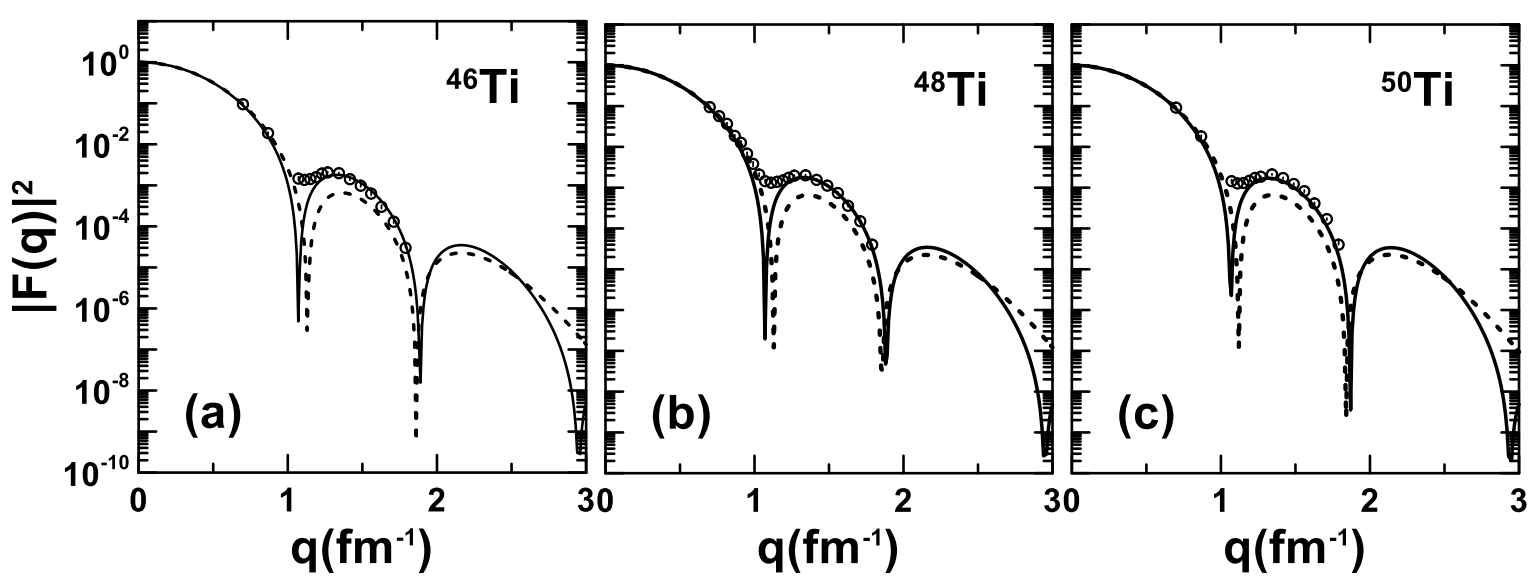

Figure 1-Elastic charge form factors for ${ }^{46,48,50} \mathrm{Ti}$-isotopes are plotted versus the momentum transfer $q$ (in $\mathrm{fm}^{-1}$ ). The dashed and solid curves are the computed form factors without and with the effect of SRCs, correspondingly. The experimental data (the symbols of open circle) for ${ }^{46} \mathrm{Ti}$ (a) and ${ }^{50} \mathrm{Ti}$ (c) is taken from [28] while that for ${ }^{48} \mathrm{Ti}$ (b), the symbols of open circle and triangle are taken from [29] and [30], correspondingly.

In Figures-(2 and 3), the ECFF's of ${ }^{52,54} \mathrm{Cr}$-isotpes and ${ }^{56,}{ }^{58} \mathrm{Fe}$-isotopes, respectively, that are computed without SRCs (labeled by the dashed line) and with considering of SRC's (labeled by the solid line) are exhibited and compared with the corresponding observed data (labeled by the symbols). Here, the occupation number of proton in $1 \mathrm{f}$ - and 2 p-orbitals is supposed to have $\eta_{1 f}=0.286$, $\eta_{2 p}=0\left[\eta_{1 f}=0.429\right.$ and $\left.\eta_{2 p}=0\right]$, correspondingly. It is evident that the observed data at $q \leq 1.1$ and $q \geq 1.8 \mathrm{fm}^{-1}\left[q \leq 0.85\right.$ and $\left.q \geq 1.7 \mathrm{fm}^{-1}\right]$ is good estimated by the dashed line, whereas at $1.1<q<1.8 \mathrm{fm}^{-1} \quad\left[0.85<q<1.7 \mathrm{fm}^{-1}\right]$, the observed data for the first minimum [second maximum] is markedly deviated [underestimated] by this line. Inclusion of the influence of SRCs (the solid line) tends to enhance the calculated results of ECFFs at $1.1 \leq q \leq 1.8 \mathrm{fm}^{-1}$ $\left[0.85<q<1.7 \mathrm{fm}^{-1}\right]$ which sequential tends to bring these ECFFs into the position of accordance with the observed data. Generally, the manner and magnitude of the solid line exhibit a good accordance with the observed data. Furthermore, all diffraction minima and maxima calculated by the solid line are in remarkable agreement with the observed data. 


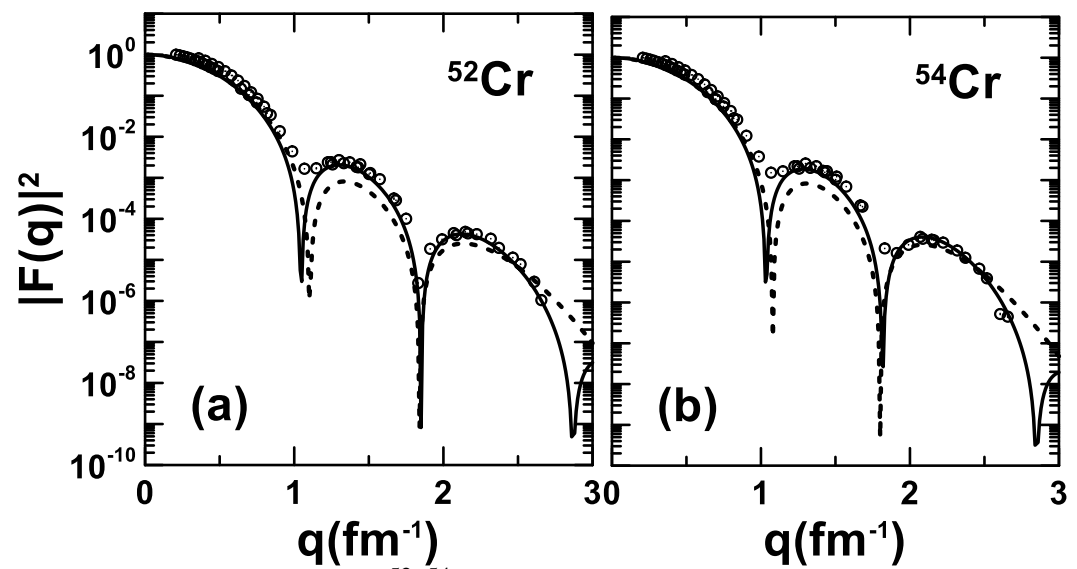

Figure 2-Elastic charge form factors for ${ }^{52,}{ }^{54} \mathrm{Cr}$-isotopes. The organization of the figure, e.g. symbols and lines, is similar to that of Fig. 1. The experimental data (designated by the open circle symbols) is taken from [31].

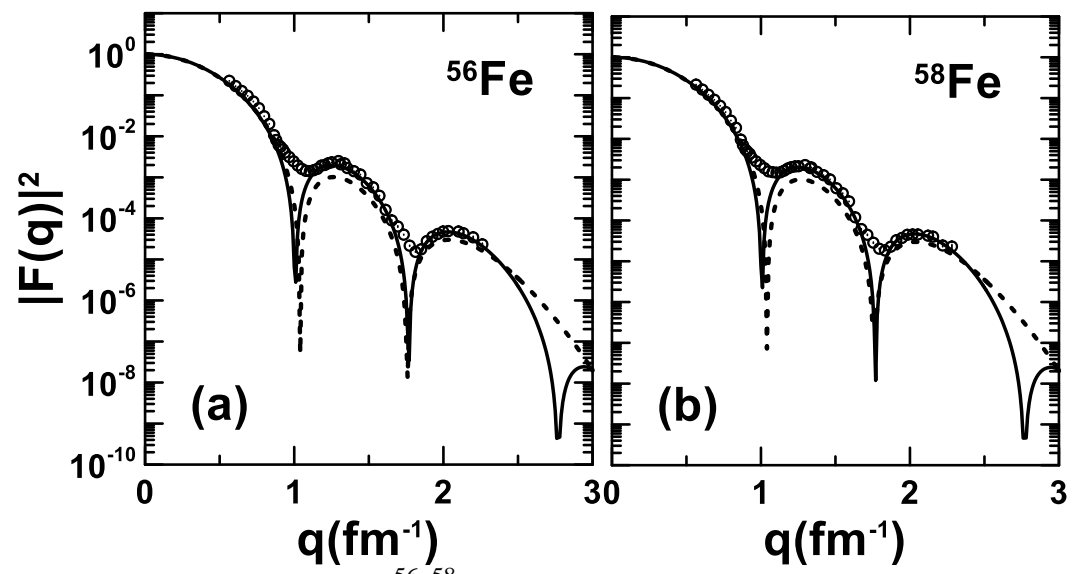

Figure 3- Elastic charge form factors for ${ }^{56,58} \mathrm{Fe}$-isotopes. The organization of the figure, e.g. symbols and lines, is similar to that of Fig. 1. The experimental data (labeled by the open circle symbols) is taken from [32].

In Figure-4, the ECFFs of ${ }^{72,74,76} \mathrm{Ge}$-isotopes computed without SRCs (referred to by the dashed line) and with SRCs (referred to by the solid line) are shown and compared with those of measured data. In these isotopes, the proton occupation number in $1 \mathrm{f}$ - and $2 \mathrm{p}$-orbitals is presumed to have $\eta_{1 f}=0.714$ and $\eta_{2 p}=0.333$, correspondingly. It is obvious that the data at $q>0.8 \mathrm{fm}^{-1}$ (along the second, third and fourth diffraction maxima) is obviously under-predicted by the dashed line.

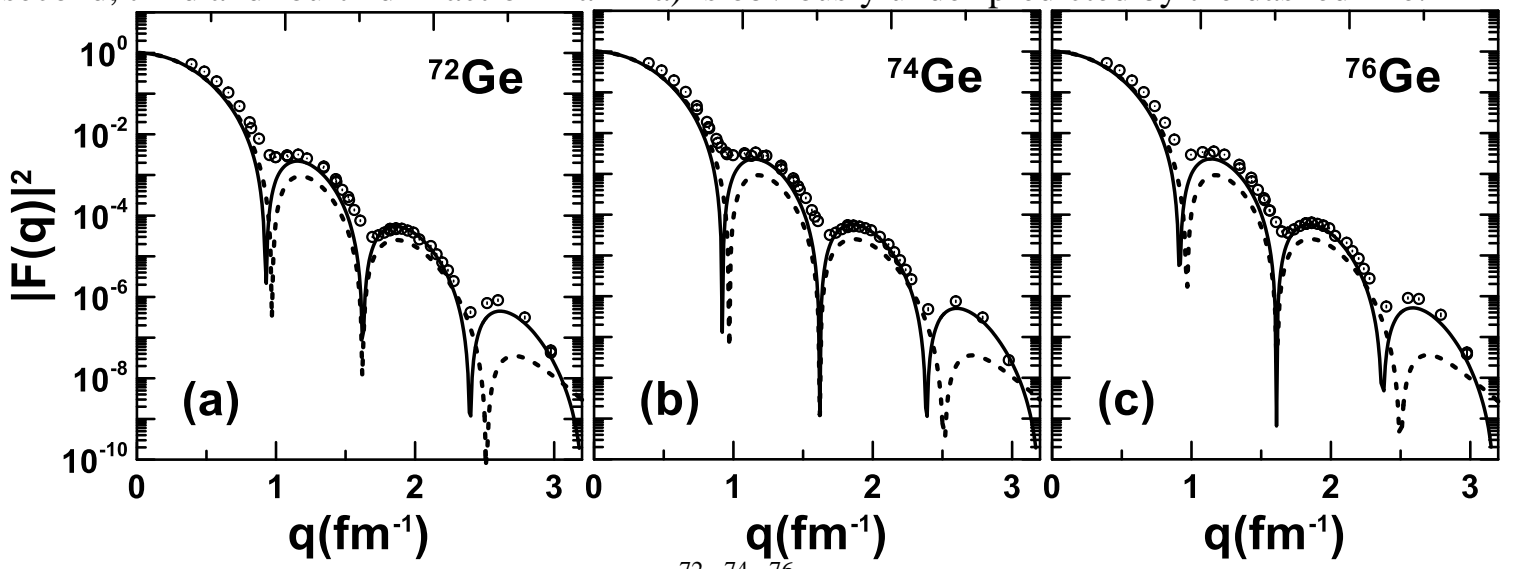

Figure 4-Elastic charge form factors for ${ }^{72,}{ }^{74},{ }^{76} \mathrm{Ge}$-isotopes. The organization of the figure, e.g. symbols and lines, is similar to that of Figure-1. The experimental data (designated by the open circle symbols) is taken from [33]. 
In addition, the first [third] diffraction minimum found in the data is slightly [markedly] deviated toward the high momentum transfer by the dashed line. Inserting the influence of SRCs modifies the calculated ECFFs (the solid line) at $q>0.8 \mathrm{fm}^{-1}$ which sequential tends to take them along the position of accordance with the data. Commonly, the presentation of the solid line reveals a very good agreement with the measured data along the whole considered $q$ values. Moreover, all measured maxima and minima are mimicked at correct places of momentum transfer axis.

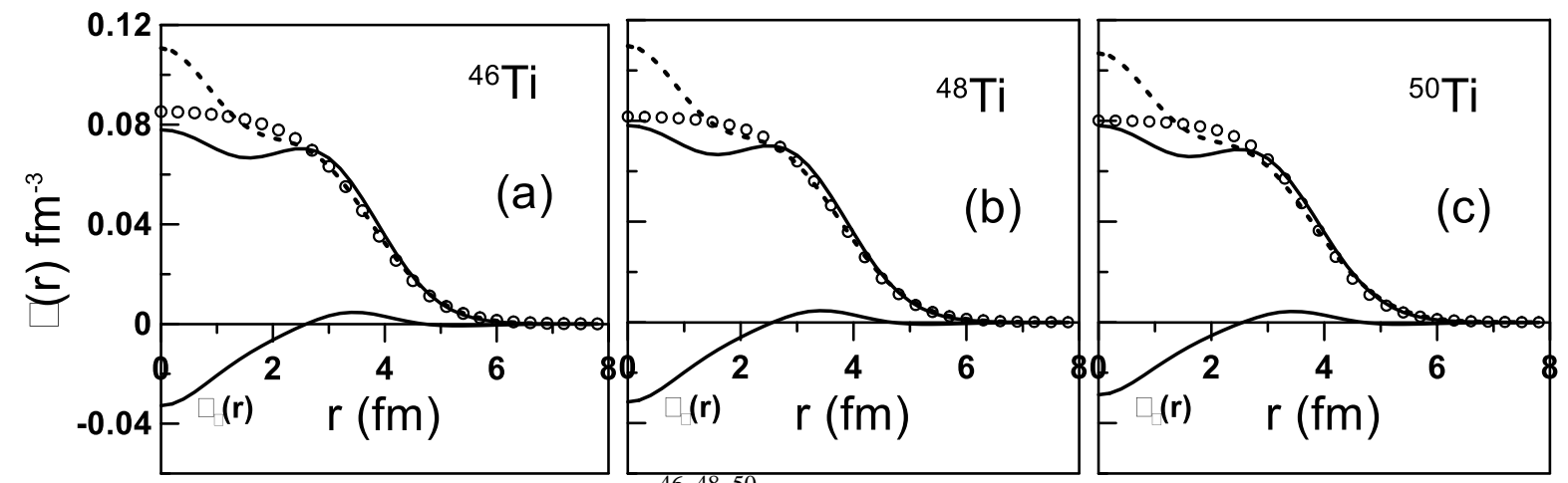

Figure 5-Charge density distributions for ${ }^{46,48,50}$ Ti-isotopes. The computed densities without the effect of SRCs (described by the dashed line) and with the effect of SRCs (described by the solid line) are exposed and compared with those fitted to the experimental ones (described by the open circle symbols) [28]. The difference between the dashed and solid distributions is labeled by the symbol $\rho_{2}(r)$.

In Figures-(5-8), the ground state CDDs, $\rho_{c h}^{g s}(r)$, of a number of fp-shell nuclei computed without SRCs (denoted by the dashed line) and with SRCs (denoted by the solid line) are exposed and compared with those fitted to the data (denoted by the open circle symbols) [26, 27]. Additionally, the partaking of SRCs

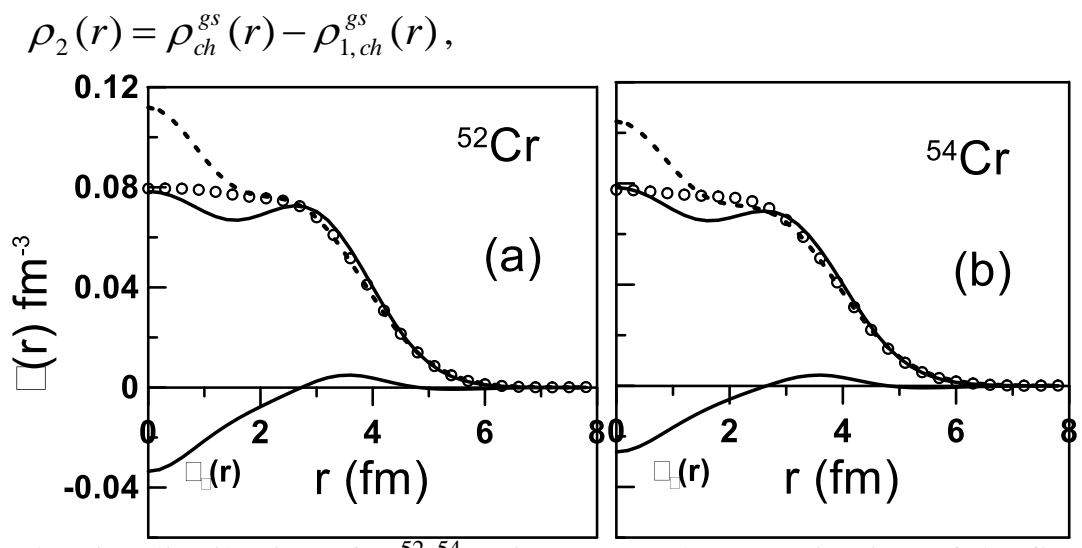

Figure 6-Charge density distributions for ${ }^{52,54} \mathrm{Cr}$-isotopes. The organization of the figure, e.g. symbols and lines, is similar to that of Figure-5. The experimental data (designated by the open circle symbols) is taken from $[26,27]$. 


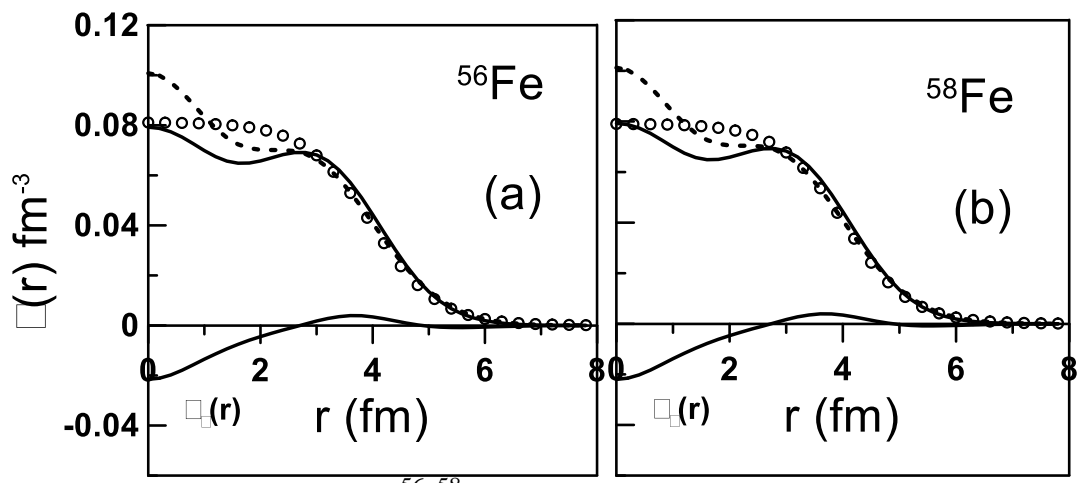

Figure 7-Charge density distributions for ${ }^{56,58} \mathrm{Fe}$-isotopes. The organization of the figure, e.g. symbols and lines, is similar to that of Figure-5. The experimental data (designated by the open circle symbols) is taken from $[26,27]$.
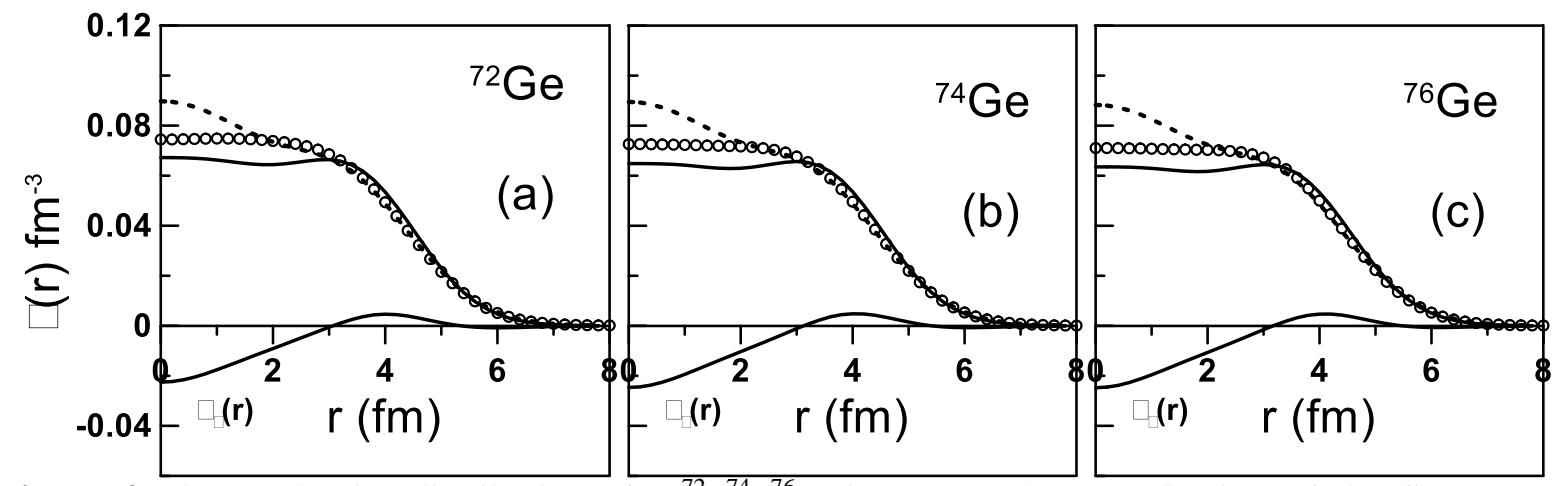

Figure 8-Charge density distributions for ${ }^{72,74,76} \mathrm{Cr}$-isotopes. The organization of the figure, e.g. symbols and lines, is similar to that of Figure-5. The experimental data (designated by the open circle symbols) is taken from previous articles [26, 27].

The $\rho_{c h}^{g s}(r)$ is also shown in the above figures and the presentation of $\rho_{2}(r)$ is described by some oscillations around the $r$-axis. It is understandable from the above figures that the possibility of a proton to exist nearby the central fragment $(0 \leq r \leq 3 \mathrm{fm})$ of $\rho_{c h}^{g s}(r)$ is greater than the tail fragment $\left(r>3 \mathrm{fm}^{-1}\right)$. Besides, taking into account the SRCs effect leads to diminish considerably the central fragment of $\rho_{c h}^{g s}(r)$ and to increase slightly the tail fragment of $\rho_{c h}^{g s}(r)$ as illustrated by the solid lines of the above figures. This means that the insertion of SRCs causes to increase the possibility of relocating the protons from the central fragment of the nucleus toward its surface which sequential causes to enlarge the rms charge radii and hence reduces the rigidity of nuclei. To keep the size of nuclei within the fixed experimental value, the parameter $b$ must be diminished when taking into account the influence of SRCs, see Table- 1. On the other hand, inclusion of SRCs leads to modify the computed distributions of $\rho_{c h}^{g s}(r)$ (solid line) and improves the degree of accordance with the data.

\section{Conclusions}

It is concluded that introducing short-range correlation effects is essential for the attainment of a remarkable adjustment in the estimated ECFFs and necessary for the spectacular description of electron scattering data over the complete range of measured $q$.

\section{References}

1. Elton L. R. B. 1961. Nuclear Sizes. Clarendon, Oxford; Uberall H. 1971. Electron Scattering from Complex Nuclei. Academic, New York, and London; Barrett R. C. \& Jackson D. F. 1977. Nuclear sizes and Structure. Clarendon, Oxford.

2. Czyz W. and Lesniak L. 1967. Scattering of high energy electrons and nucleons from ${ }^{4} \mathrm{He}$ and nucleon-nucleon correlations, Physics Letters, B25: 319-321. 
3. Khana F. C. 1968. Nuclear structure of ${ }^{40} \mathrm{Ca}$ and elastic scattering of $750-\mathrm{MeV}$ electrons. Physical Review Letters, 20: 871-873

4. Ciofi C. and Atti degli. 1969. Elastic scattering of high-energy electrons and correlation structureof light nucli. Nuclear Physics, A129: 350-368

5. Ciofi C., Atti degli and Kabachnik N. M. 1970. Short range correlations and high energy electron scattering. Physics Review, C1: 809.

6. Ripka G. and Gillespie J. 1970. Effect of the Jastrow Correlation Factor on Nuclear Charge Distributions. Physical Review Letters, 25: 1624-1625

7. Gaudin M., Gillespie J. and Ripka G. 1971. Jastrow correlations. Nuclear Physics, A176: 237-260

8. Bohigas $O$. and Stringari S. 1980. Effects of short range correlations on nuclear mass and momentum distributions. Physics Letters, B95: 9-12

9. Dal Ri M., Stringari S. and Bohigas O. 1982. Effects of short range correlations on one- and twobody properties of nuclei. Nuclear Physics, A376: 81-93

10. Nassena H. P. 1981. The factor cluster expansion model operator approach and the charge form factor of the 4He nucleus. Journal of Physics G: Nuclear Physics, 7: 927-936.

11. Stoitsov M. V., Antonov A. N. and Dimitrova S. S. 1993. Short-range correlations and one-body properties of nuclei . Z. Phys. A345: 359-372; Natural orbital representation in nuclei. Physical Review C47: R455; Natural orbital representation and short-range correlations in nuclei. Physical Review C48: 74-86

12. Grypeos M. E. and Ypsilantis K. N. 1989. A single-particle model momentum distribution for 4He. J. Phys. G15: 1397 ; Ypsilantis K. N. \& Grypeos M. E. 1993 Proceedings of the $4^{\text {th }}$ Hellenic Symposium on Nuclear Physics (Ioannina, Greece); Grypeos M. E. \& Ypsilantis K. N. 1995. The nucleon momentum distribution in light nuclei J. Phys G21: 1701 (1995)

13. Massen S. E., Nassena H. P. and Panos C. P. 1988. A formula for the charge form factor of closed-shell nuclei and its application to ${ }^{16} \mathrm{O}$. Journal of Physics G: Nuclear Physics, 14: 753-764

14. Massen S. E. and Panos C. P. 1989. An approximate treatment of the correlated charge form factor of light nuclei. Journal of Physics G: Nuclear Physics, 15: 311-319

15. Massen S. E. 1990. Correlated charge form factor and densities of the s-d shell nuclei. Journal of Physics G: Nuclear Physics, 16: 1713-1725

16. Jastrow R. 1955. Many-Body Problem with Strong Forces. Physical Review, 98: 1479-1584

17. Clark J. W. and Ristig M. L. 1970. Cluster-Expansion Procedures for the Correlated Charge Form Factor. Nuovo Cimento, A70 313-322

18. Ristig M. L., Ter Low W. J. and Clark J. W. 1971. Tensor Correlations in Nuclear Matter Physical. Review, C3: 1504-1513.

19. Clark J. W. 1979. Variational theory of nuclear matter. Prog. Part. Nucl. Phys. 2: 89.

20. Massen S. E. and Moustakidis Ch. C. 1999. Systematic study of the effect of short range correlations on the form factors and densities of s-p and s-d shell nuclei . Physical Review, C60: 024005.

21. Sharrad F. I., Hamoudi A. K., Radhi R. A., Abdullah H. Y., Okhunov A. A. and Abu Kassim H. 2013. Elastic Electron Scattering from Some Light Nuclei. Chinese Journal of Physics, 51: 452465

22. Sharrad F. I., Hamoudi A. K., Radhi R. A. and Abdullah H. Y. 2013. Inelastic electron scattering from light nuclei J. Natn. Sci. Foundation Sri Lanka, 41: 209-217

23. Roy R. R. and Nigam B. P. 1967. Nuclear Physics theory and experiment (John Wiley \& Sons, New York)

24. de-Shalit A. and Talmi I. 1963. Nuclear Shell Theory (Academic, New York).

25. Brown B .A., Radhi R. A. and Wildenthal B.H. 1983. Electric quadrupole and hexadecupole nuclear excitations from the perspectives of electron scattering and modern shell-model theory. Physics Reports, 101: 313 - 358

26. De Vries H., De Jager C. W. and De Vries C. 1987. Nuclear charge density distribution parameters from elastic electron scattering. Atomic Data and Nuclear Data Tables, 36: 495-536

27. Fricke G. and Bernhardt C. 1995. Nuclear ground state charge radii from electromagnetic interactions . Atomic Data and Nuclear Data Tables, 60 177-285. 
28. Heisenberg J., Hofstadter R., McCarthy J. S., Herman R., Clark B. C. and Ravenhall D. Q. 1972. Electron-Scattering Determination of Isotopic Differences in the Charge Distributions of ${ }^{46,48,50} \mathrm{Ti}$. Physical Review, C6: 381-384.

29. Frosch R. F., Hofstadter R., McCarthy J. S., Nöldeke G. K., van Oostrum K. J., Yearian M. R., Clark B. C., Herman R. and Ravenhall D. G. 1968. Electron Scattering Studies of Calcium and Titanium Isotopes. Physical Review, 174: 1380.

30. Shevchenko N. G., Gonchar V. Yu., Buki A. Yu., Kasatkin Yu. A., Polishchuk V. N., Mazan'ko B. V. and Lishenko L. G. 1978. Distribution of charge density in ${ }^{48} \mathrm{Ti}$ and ${ }^{50} \mathrm{Cr}$. Soviet Journal of Nuclear Physics, 28(3): 295-297.

31. Lightbody J. W., Jr., Bellicard J. B., Cavedon J. M., Frois B., Goutte D., Huet M., Leconte Ph., Nakada A., Phan Xuan Ho, Platchkov S. K., Turck-Chieze S., de Jager C. W., Lapikás J. J. \& de Witt Huberts P. K. A. 1983. Elastic and inelastic electron scattering from ${ }^{50,52,54} \mathrm{Cr}$, Physical Review, C 27: 113.

32. Wohlfahrt H. D., Schwentker O., Fricke G., Andresen H. G. and Shera E. B. 1980. Systematics of nuclear charge distributions in the mass 60 region from elastic electron scattering and muonic $\mathrm{x}$ ray measurements, Physical Review, C22: 264.

33. Mallot G. K. 1984. Electron Scattering of Germanium and Muonic atoms of Krypton as Contribution to the Systemic of the Nuclear Charge Densities. Ph. D. thesis, University of Mainz. 